\title{
Gender Differences in the Effect of Ascorbic Acid against petroleum fume-induced Oxidative Stress and Reproductive Toxicity in Rats
}

\author{
Christopher Edet Ekpenyong* \\ Department of Physiology, University of Uyo, Nigeria
}

*Corresponding author: Christopher Edet Ekpenyong, Department of

Physiology, Faculty of Basic Medical Sciences, University of Uyo, Uyo, Nigeria.

Received Date: October 14,2020

Published Date: November 17, 2020

\begin{abstract}
Background: Biological factors affecting the therapeutic doses of ascorbic acid (AA) against xenobiotic-induced oxidative stress (OS) and reproductive toxicity have been established, however, the effect of gender is yet to be thoroughly researched and ascertained. The present study aimed to assess gender disparities in the effect of AA against gasoline vapor (GV)-induced reproductive toxicity in rats.

Methods: Thirty-five matured male and female Wistar Albino rats weighing between 200 and $250 \mathrm{~g}$ were divided into 5 groups (n=7per group). Group 1 served as unexposed control, groups 2,3, 4, and 5 were exposed to GV for 6 weeks. Groups 3, 4, and 5 in addition to being exposed to GV were treated with low, medium, and high doses of AA for 2 weeks of the 6 weeks of exposure and treatment. Animals were sacrificed and blood samples and reproductive organs were obtained for analysis and histopathological examination respectively.

Results: Exposure to GV alone significantly $\mathrm{P}<0.05$ decreased serum estrogen, progesterone, and testosterone levels. Serum levels of estrogen and progesterone were significantly $(\mathrm{P}<0.05)$ higher in the low-dose $\mathrm{AA}$-treated female animals, whereas the highest serum level of testosterone was found in the high-dose AA treated male animals. A corresponding significant decrease in serum FSH and LH levels were also found in the low and high doses of AA treated female and male groups respectively.

Conclusion: There is a gender difference in the effect of AA against GV-induced OS and reproductive toxicity. Therefore, gender-related dose adjustment should be considered when using AA to manage OS-related male or female reproductive disorders.
\end{abstract}

Keywords: Petroleum fume; Vitamin C; Gender; Oxidative stress; Reproduction; Toxicity

\section{Introduction}

Gasoline consists of several hydrocarbons and additives that constitute significant environmental pollutants. Exposure to hydrocarbon fumes by humans is common and widespread due to the extensive domestic and industrial applications. Exposure by humans can be through dermal, inhalation, or ingestion routes, with inhalation being the most common exposure route and can occur at any point along the production and distribution chain. Many people are exposed to petroleum fumes daily, especially those whose residence or workplace is close to petrochemical industries, refineries, oil fields, gasoline refueling stations, traffic-

congested areas and gasoline combustion stations. About 100 million people are exposed to hydrocarbon constituents of gasoline per week when refueling at a self-service gasoline station [1]. Also, gasoline service station attendants are at a higher risk of exposure to gasoline inhalation for many hours a week and about 8086 minutes per year. Available data indicate that $>3.6$ billion gallons of gasoline vaporize into the air as gasoline vapor (GV) [2]. Following inhalation, GV is absorbed and distributed in the body. Within the body, it undergoes further toxicokinetic processes leading to the generation of reactive oxygen species (ROS), oxidative stress (OS), 
and damage to tissues with resultant altered tissue structures and function. Other pathophysiological processes contributing to tissue damage following exposure to GV include inflammation and immune system dysfunction. Previous studies indicate that gasoline constituents, especially benzene, ethylbenzene, toluene, and xylene (BTEX compounds), are particularly toxic to the reproductive system, hence are classified as reproductive toxicants. Reproductive system risk assessment indicates that exposure to GV can cause a wide array of male reproductive system disorders including, decreased sperm count, sperm motility, the volume of ejaculation, abnormal sperm morphology, and damage to sperm deoxyribonucleic acid. Furthermore, there is an increased serum follicle-stimulating hormone (FSH) and luteinizing hormone (LH). Also reported are menstrual disorders and several obstetric and gynecological disorders. Studies have also shown that these adverse reproductive effects could be attenuated by supplementation with synthetic antioxidant vitamins such as ascorbic acid (AA). A consideration of the existing literature suggests that an influence of AA against xenobiotic-induced reproductive toxicity relates to its ability to attenuate oxidative stress (OS) [3,4]. Evidence is emerging that the effect of AA in human subjects can be beneficial or harmful depending on different biological factors such as species, duration of exposure, age, body mass index, and lifestyle habits $[5,6]$. However, no evidence has emphasized the effect of gender on the therapeutic outcomes of AA in both physiological and pathological states. Possible gender differences are of toxicology concern because of gender differences in anatomy and physiological control mechanisms for reproduction. Also, gender is known to affect the initiation and progression of OS and immune system dysfunctionmediated disorders. Recent studies have demonstrated gender dimorphism in OS and immune responsiveness and susceptibility to endocrine and reproductive disorders. The present study aimed to evaluate gender differences in the effect of AA against GV-induced OS and reproductive toxicity in rats.

\section{Materials and Methods}

\section{Experimental Animals}

Thirty-five mature Wistar Albino rats weighing between 200 and $250 \mathrm{~g}$ were obtained from the animal house of the Department of Pharmacology, Faculty of Pharmacy, University of Uyo, Akwa Ibom State, Nigeria. They were kept in well-ventilated cages for 7 days to acclimatize. They were allowed access to food and water ad libitum. All animals were fed rat chow (Vital Feeds, Grand Cereal Ltd, Jos).

\section{Segregation of Animals}

The animals were randomly divided into 5 groups $(n=7$ per group). They were exposed to GV in the exposure chambers (60 $\mathrm{x}$ $80 \times 100 \mathrm{~cm} 3$ )

Group 1 served as unexposed control and was orally gavaged $2 \mathrm{ml}$ of normal saline for $6 \mathrm{wks}$.
Group 2 was exposed to GV alone for 6 wks and maintained on normal animal feed.

Group 3 was exposed to GV for 6 wks, fed a normal diet, and orally gavaged $100 \mathrm{mg} / \mathrm{kg}$ of AA for $2 \mathrm{wks}$ of the $6 \mathrm{wks}$.

Group 4 was exposed to GV for 6 wks, fed a normal diet, and orally gavaged $200 \mathrm{mg} / \mathrm{kg}$ of AA for $2 \mathrm{wks}$ of the $6 \mathrm{wks}$.

Group 5 was exposed to GV for 6 wks, fed a normal diet, and orally gavaged $300 \mathrm{mg} / \mathrm{kg}$ of AA for $2 \mathrm{wks}$ of the $6 \mathrm{wks}$.

\section{Collection of experimental samples for analysis}

After 2 weeks of AA administration, the animals were weighed and anesthetized with chloroform soaked in a swab of cotton wool in a desiccator. The blood sample was collected by cardiac puncture and emptied into labeled specimen bottles, for biochemical evaluation including determination of estrogen, progesterone, follicle-stimulating hormone (FSH), Luteinizing hormone ( $\mathrm{LH})$, testosterone level, catalase (CAT), and malondialdehyde (MDA) levels. Animals were sacrificed by cervical dislocation and reproductive organs testis and ovary were carefully removed and fixed in a suitably treated formalin reagent and thereafter, subjected to normal routine histological procedures/examination.

\section{Biochemical Analysis}

\section{Estimation of CAT and MDA activities}

CAT activity was determined by the Titrimetric method. Tissue lipid peroxidation was quantified by estimating the plasma concentration of MDA using the thiobarbiturate acid reactive substance (TBARS) method and measured spectrophotometrically at 532nm. Serum estrogen, progesterone, testosterone, LH, and FSH levels were determined by Enzyme-Linked Immuno-sorbent assay (ELISA) as described by Tietz [7].

\section{Histopathological tissue processing}

The fixed tissues were dehydrated in different grades of alcohol as follows; two changes of $70 \%$ and $95 \%$ alcohol for a period of $2 \mathrm{hrs}$ each, two changes of $100 \%$ also known as absolute alcohol for a period of $2 \mathrm{hrs}$. Dehydrated tissues were cleared using xylene. Tissues were impregnated with two changes of paraffin wax in the oven at the temperature of $60^{\circ} \mathrm{C}$ for $1 \mathrm{hr} 30 \mathrm{mins}$ ) each to ensure they were fully embedded. Tissues were transferred from the final wax bath to molds filled with molten wax, allowed to solidify and thereafter, properly oriented for sectioning. The paraffin block was sectioned at $5 \mu \mathrm{m}$ after cooling the surface of the tissues with an ice bar. Ribbons were gently picked with Carmel brush and dropped in a water bath containing water at $60^{\circ} \mathrm{C}$ to enable ribbons float, expand and flatten out. Slides were rubbed with thymol containing egg albumen and gently dipped into the bath to pick up the flattened out tissue ribbons [8]. Haematoxylin and Eosin (H\&E) staining techniques [8] were applied in staining the tissue sections. 


\section{Haematoxylin and eosin staining procedures}

Tissue sections were deparaffinized in two changes of xylene and hydrated through graded series of alcohols in descending order and were rinsed in water and stained with Haematoxylin for $10 \mathrm{mins}$. Tissue sections were rinsed and differentiated in one percent (1\%) acidic alcohol and blued in running water using saturated lithium carbonate solution until sections appear sky blue. The blued section was counterstained in the Eosin solution for 3 mins. Tissues were washed in water and dehydrated in ascending grades of alcohol, cleared in xylene, and mounted in DPX covered with coverslips and observed under the microscope.

\section{Microscopy}

Processed slides were viewed under a light microscope at magnification (X400), and photomicrographs obtained were linked to the computer using the microscope's camera.

\section{Statistical Analysis}

Statistical analysis was carried out using Statistical Package for
Social Sciences (SPSS), version 20.0, and M. S. Excel. The one-way analysis of variance (ANOVA) and posthoc Tukey least significant difference (LSD) test was used to analyze the data and to determine the significance respectively. Data are expressed as Mean + Standard Error of Mean (S.E.M) and tables were used to illustrate the variations in the numerical values across the experimental groups. The P. values $<0.05$ were considered statistically significant.

\section{Results}

\section{Antioxidant activity}

Serum MDA increased significantly in GV alone group and decreased significantly $(\mathrm{P}<0.05)$ in a dose-independent manner in both male and female animals. In female animals, serum MDA significantly $(\mathrm{P}<0.05)$ increased in GV alone group, and significantly $(\mathrm{P}<0.05)$ decreased in GV plus low dose AA compared to the gasoline alone, group. Interestingly, serum CAT decreased significantly $(\mathrm{P}<0.05)$ in GV plus a medium and high dose of AA compared with the GV alone group (Figures 1-4).

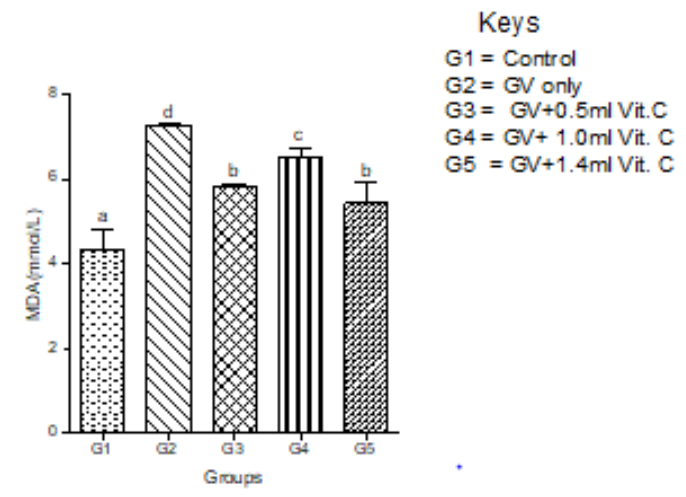

Figure 1: Effect of AA on GV-induced changes in serum MDA levels in male animals. Values are expressed as Mean $\pm \mathrm{SEM}$ at $\mathrm{P}<0.05$ relative to control. Different letters indicate significance while similar letters represent non-significance.

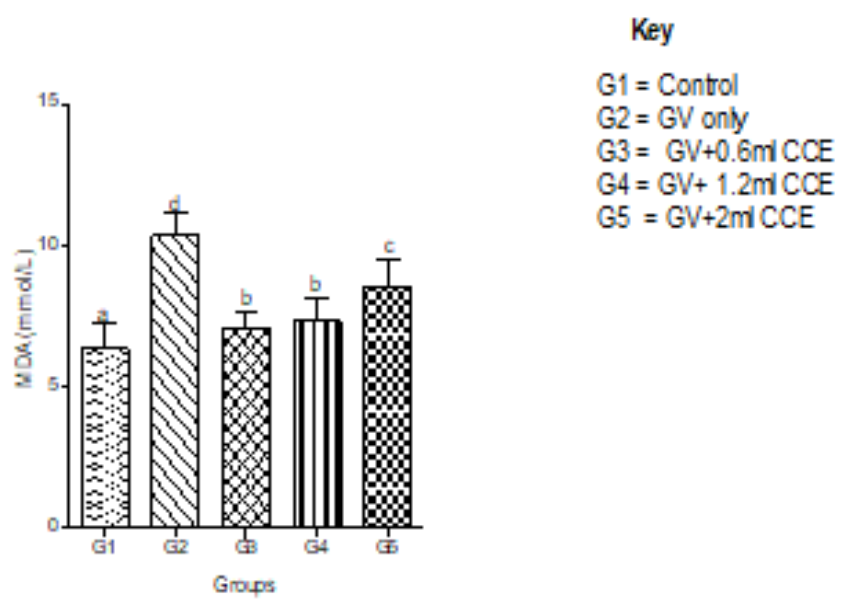

Figure 2: Effect of AA on GV-induced changes in serum MDA levels in female animals. Values are expressed as Mean $\pm \mathrm{SEM}$ at $\mathrm{P}<0.05$ relative to control. Different letters indicate significance while similar letters represent non-significance. 


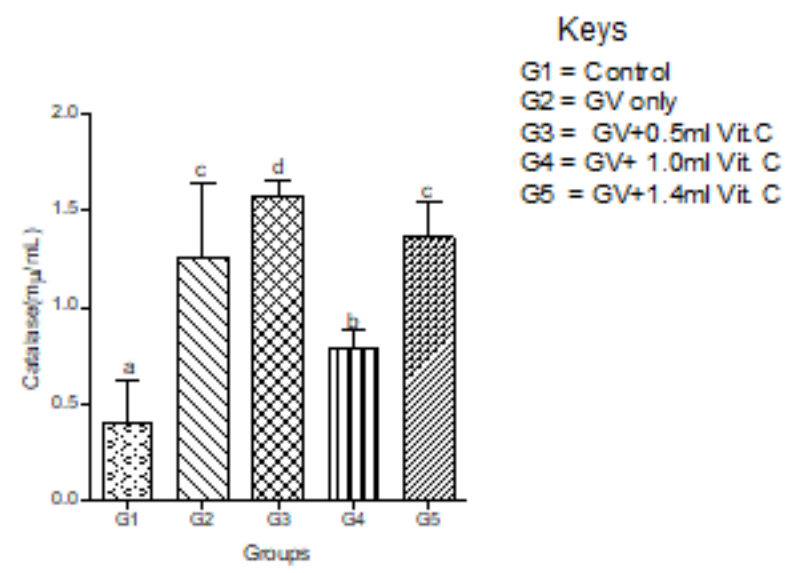

Figure 3: Effect of AA on GV-induced changes in serum catalase in male animals. Values are expressed as Mean $\pm \mathrm{SEM}$ at $\mathrm{P}<0.05$ relative to control. Different letters indicate significance while similar letters represent non-significance.

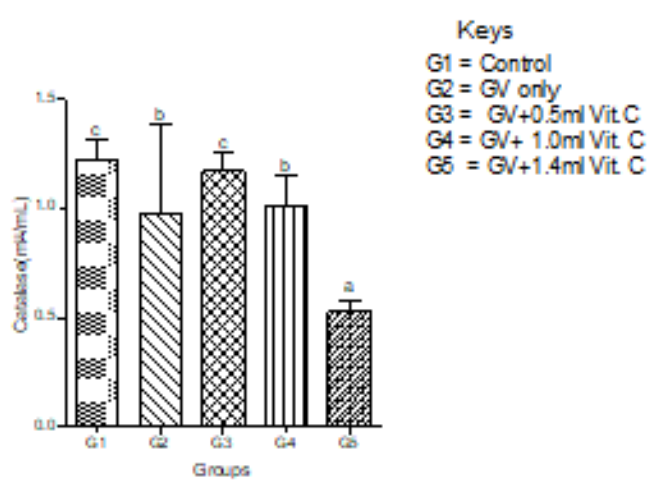

Figure 4: Effect of AA on GV-induced changes in serum catalase in female animals. Values are expressed as Mean $\pm S E M$ at $P<0.05$ relative to control. Different letters indicate significance while similar letters represent non-significance.

\section{Serum estradiol level}

Means serum level of estradiol significantly $(\mathrm{P}<0.05)$ decreased in the GV-alone group compared with the control group. Estradiol significantly increased in GV plus low, medium, and high doses compared with the GV-alone group. The highest increment occurred in the group treated with low-dose of AA (Figure 5).

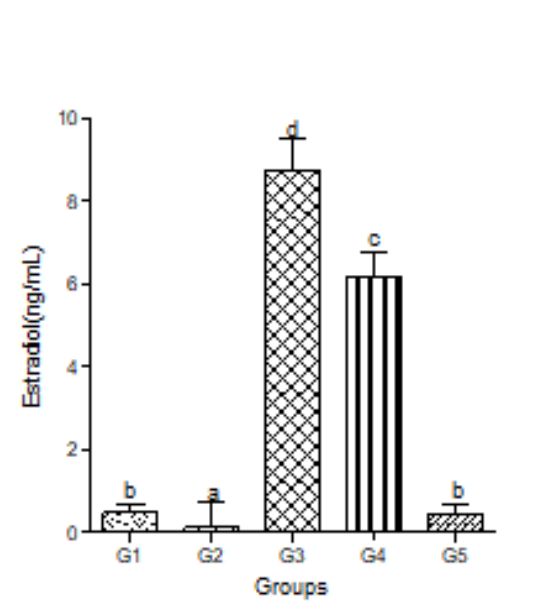

Keys

$\mathrm{G} 1=$ Control

$\mathrm{G} 2=\mathrm{GV}$ only

$\mathrm{G} 3=\mathrm{GV}+0.5 \mathrm{ml}$ Vit. $\mathrm{C}$

$\mathrm{G} 4=\mathrm{GV}+1.0 \mathrm{mI}$ Vit. $\mathrm{C}$

$\mathrm{G} 5=\mathrm{GV}+1.4 \mathrm{mI}$ Vit. $\mathrm{C}$

Figure 5: Effect of AA on GV-induced changes in serum Estradiol level. Values are expressed as Mean \pm SEM at $\mathrm{P}<0.05$ relative to control. Different letters indicate significance while similar letters represent non-significance. 


\section{Serum follicle-stimulating hormones (FSH) level}

Exposure of the female animals to GV-alone caused a significantly $(\mathrm{P}<0.05)$ decreased in serum FSH compared with normal control, whereas in male animals exposed to GV had a non- significant $(\mathrm{P}<0.05)$ changes in serum FSH compared with normal control. Also, the highest ameliorative effect of AA was obtained from the highest dose in female whereas, in the male animals, it was the medium dose that produced the highest ameliorative effect (Figure 6,7).

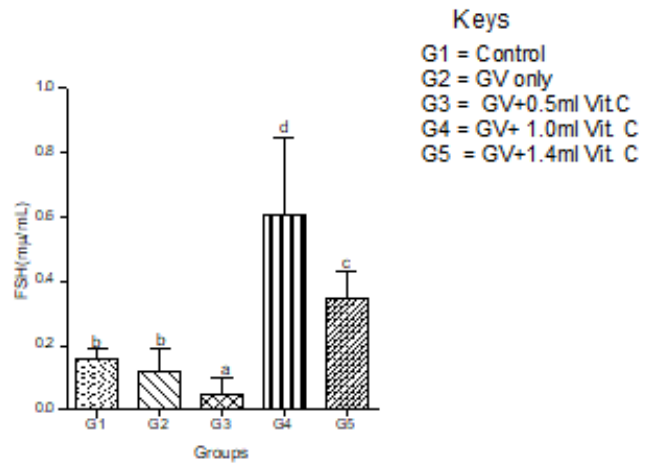

Figure 6: Effect of AA on GV-induced changes in serum FSH in male animals. Values are expressed as Mean \pm SEM at $\mathrm{P}<0.05$ relative to control. Different letters indicate significance while similar letters represent non-significance.

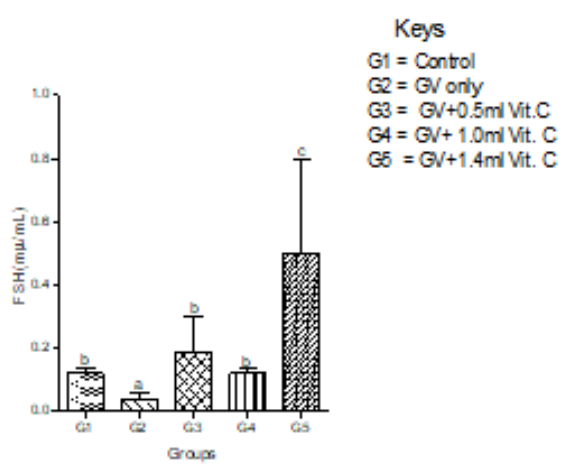

Figure 7: Effect of AA on GV-induced changes in serum FSH in female animals. Values are expressed as Mean \pm SEM at $\mathrm{P}<0.05$ relative to control. Different letters indicate significance while similar letters represent non-significance.

\section{Serum luteinizing hormone (LH) level}

In female animals, there was no significant $(\mathrm{P}>0.05)$ difference between the serum level of $\mathrm{LH}$ between the GV alone and the normal control. In the male animals, a significant $(\mathrm{P}<0.05)$ decrease in serum LH compared with the normal control was observed. In both sexes, medium doses of AA produced the highest protective effect on serum LH (Figure 8,9).

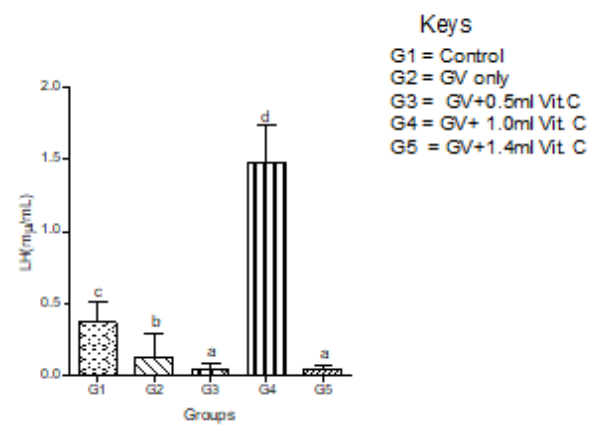

Figure 8: Effect of AA on GV-induced changes in serum LH in male animals. Values are expressed as Mean $\pm \mathrm{SEM}$ at $\mathrm{P}<0.05$ relative to control. Different letters indicate significance while similar letters represent non-significance. 


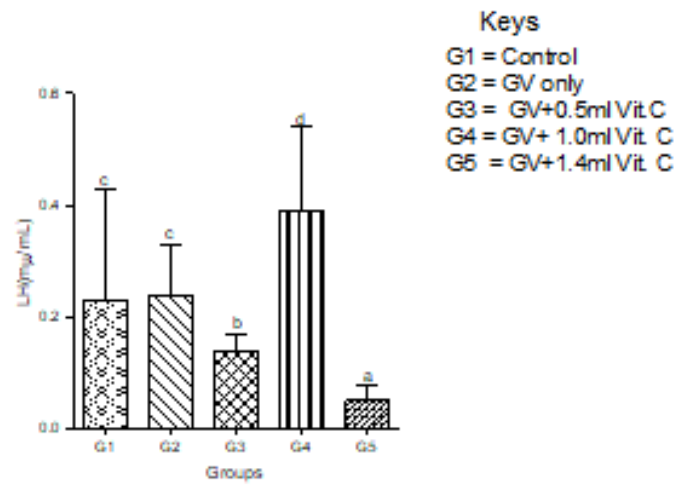

Figure 9: Effect of AA on GV-induced changes in serum LH in female animals. Values are expressed as Mean \pm SEM at $P<0.05$ relative to control. Different letters indicate significance while similar letters represent non-significance.

\section{Serum progesterone level}

Exposure to GV significantly $(\mathrm{P}<0.05)$ decreased in serum progesterone low dose of AA produced a significantly increased in serum progesterone level compared with normal control. Medium and high doses caused a significant $(\mathrm{P}<0.05)$ decrease in serum progesterone (Figure 10).

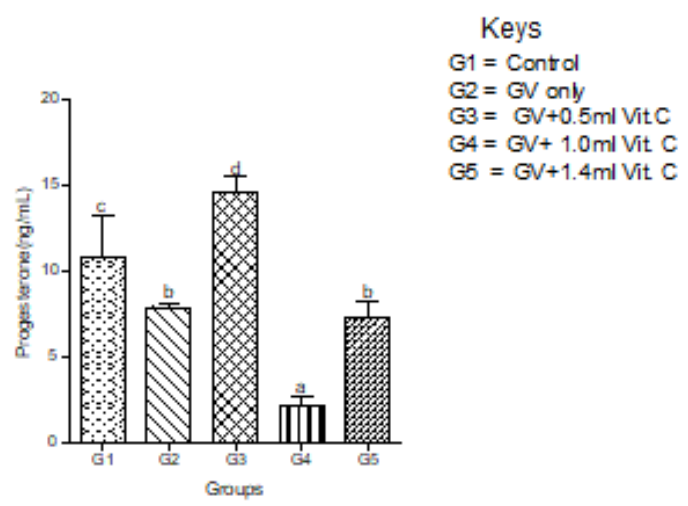

Figure 10: Effect of AA on GV-induced changes in serum Progesterone in female animals. Values are expressed as Mean $\pm \mathrm{SEM}$ at $\mathrm{P}<0.05$ relative to control. Different letters indicate significance while similar letters represent non-significance.

\section{Serum testosterone level}

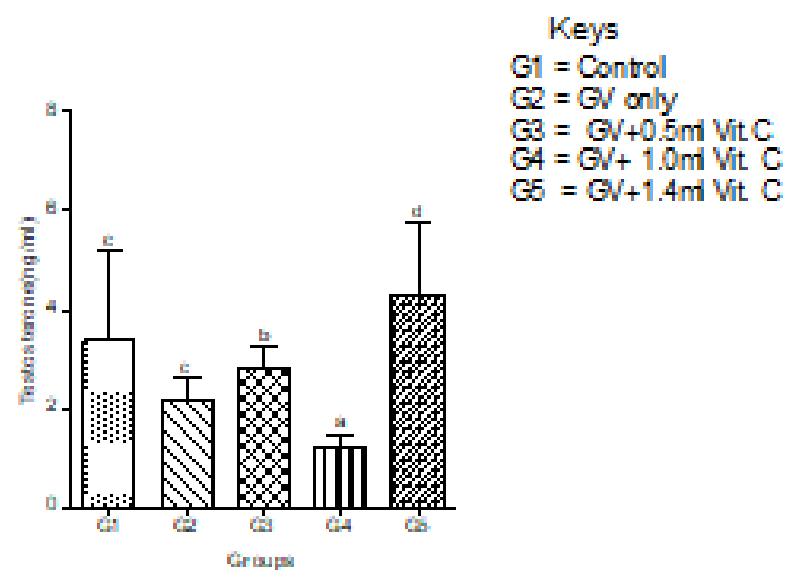

Figure 11: Effect of AA on GV-induced changes in serum testosterone. Values are expressed as Mean $\pm \mathrm{SEM}$ at $\mathrm{P}<0.05$ relative to control. Different letters indicate significance while similar letters represent non-significance. 
Exposure to GV caused a non-significant decrease in serum testosterone levels compared to levels in the normal control group. The highest dose of AA produced the highest ameliorative effect on the GV-induced decrease in serum testosterone levels (Figure 11).

Figures 12 to 26 show the changes in the histomorphology of the ovaries (Figures 12-16), testis (Figures 17-21), and epididymis
(Figures 22-25) following the exposure to GV, and concomitantly treated with AA. Similar to the changes in the biochemical markers of ovarian and testicular endpoints, a greater improvement in the ovarian histomorphology was found in the low dose AA-treated group, whereas the testicular and epididymal histomorphology showed greater improvement in the high dose AA-treated groups (Figure 12-26).

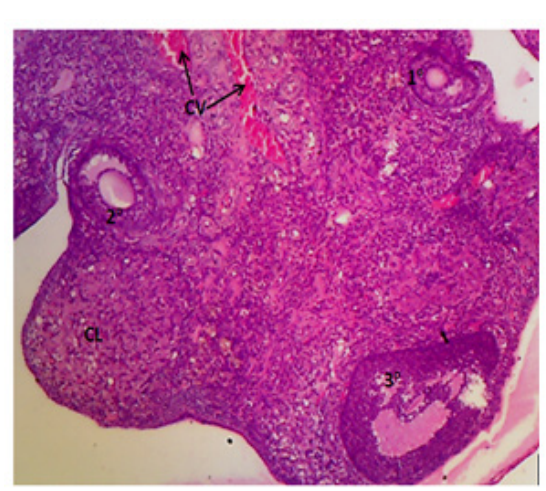

Figure 12: A section of the Ovary: (Control Group) Normal histology architecture of the ovary(H \& E Stain) at magnification (X400) showing developing follicles of varying stages and sizes (Primary, Secondary, and Tertiary follicles), and corpus luteum (CL). The stroma is pervaded with dilated congested blood vessels (CV).

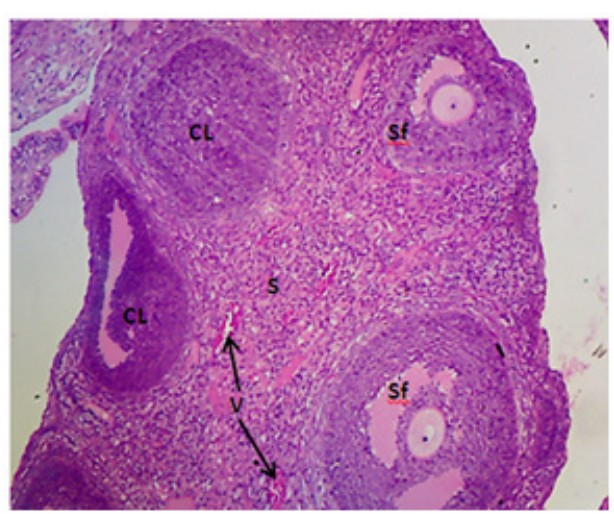

Figure 13: A section of the Ovary: (Gasoline only group) Normal histology architecture of the ovary(H \& E Stain) at magnification (X400) showing early secondary follicles (Sf), corpus luteum $(\mathrm{CL})$ and Stroma $(\mathrm{S})$ with congested blood vessels $(\mathrm{v})$.

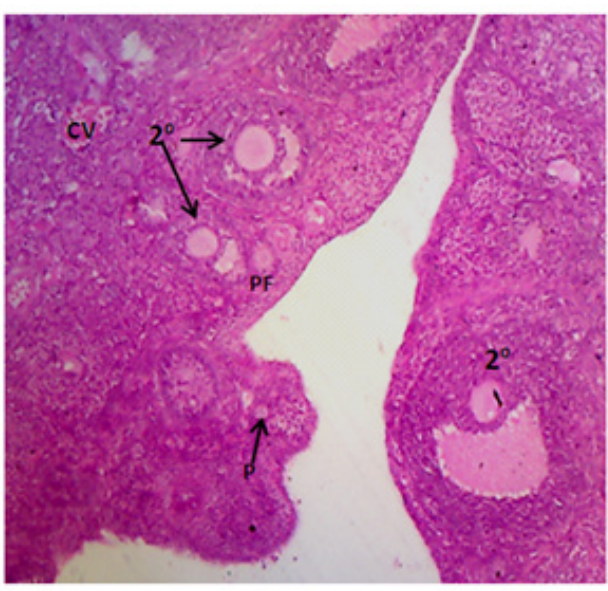

Figure 14: A section of the Ovary: (low Dose AA group) Normal histology architecture of the ovary(H \& E Stain) at magnification (X400) showing developing secondary follicles (2o), Primary follicle (Pf), and primordial follicles (P) and blood vessels (v). 


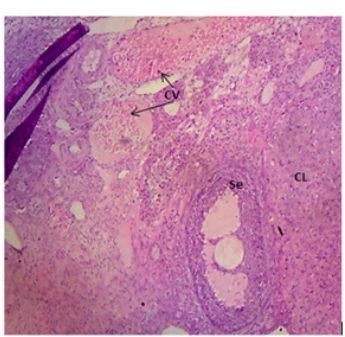

Figure 15: A section of the Ovary: (Medium Dose AA group) Normal histology architecture of the ovary(H \& E Stain) at magnification (X400) with developing secondary follicle (20), corpus luteum $(\mathrm{CL})$ and dilated congested blood vessels $(\mathrm{CV})$.

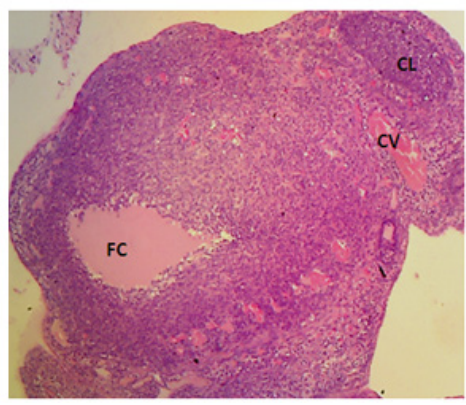

Figure 16: A section of the Ovary: (High Dose AA group) A section of the ovary (H \& E Stain) at magnification (X400) showing matured follicular cavity $(F C)$, corpus lutuem $(C L)$, and several congested blood vessels.

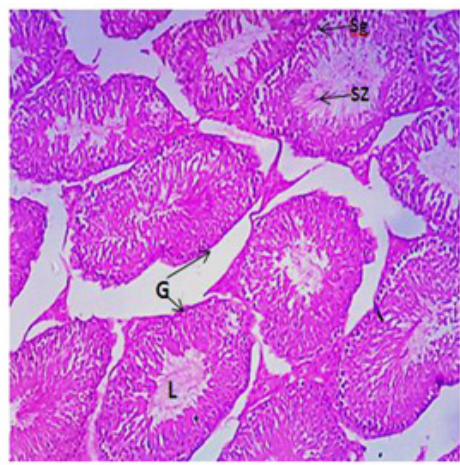

Figure 17: A section of the Testis (Control Group) A section of the testis (H \& E Stain) at magnification (X400) showing normal testicular architecture and normal size seminiferous tubules with germinal cell layers composed of spermatogonia (Sg) and spermatogenesis progressing from the basal germinal layer $(G)$ to mature spermatozoa $(S z)$ with their tails extending into the lumen $(L)$.

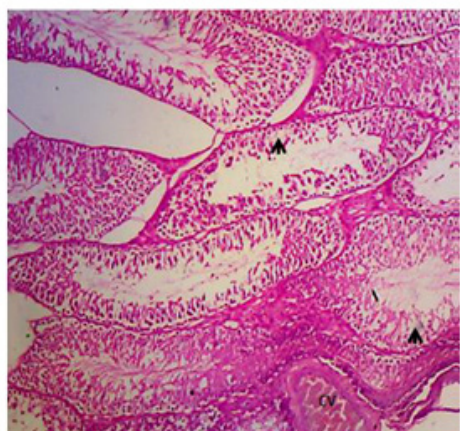

Figure 18: A section of the Testes (GV-only group) Histological section of the Testis (H-E Stain), at magnification (X400) showing testicular cytoarchitecture with multiple foci areas of degenerated seminiferous tubules, depleted germ cells (arrowheads), and congested blood vessels (CV) between the tubules. The epididymal tissue shows decrease volume within the lumen (L). The clear area $(\mathrm{H})$ indicates a reduction in spermatozoa within the lumen. 


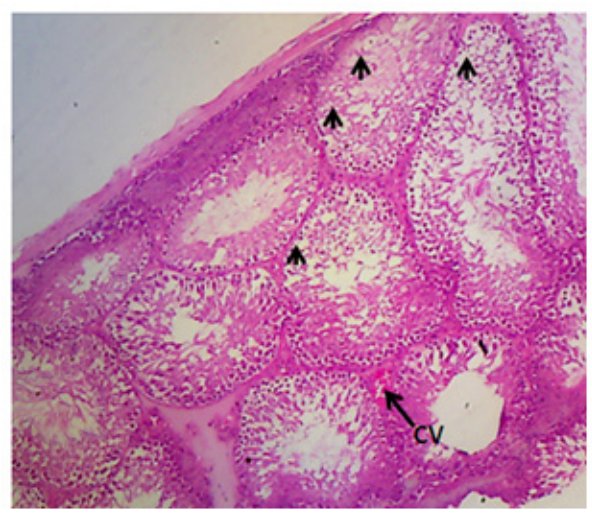

Figure 19: A section of the Testes (low dose AA group) A section of the Testes (H \& E Stain) at magnification (X400) showing multiple foci of varying degree of degenerated seminiferous tubules (arrow heads) and congested blood vessels (CV).

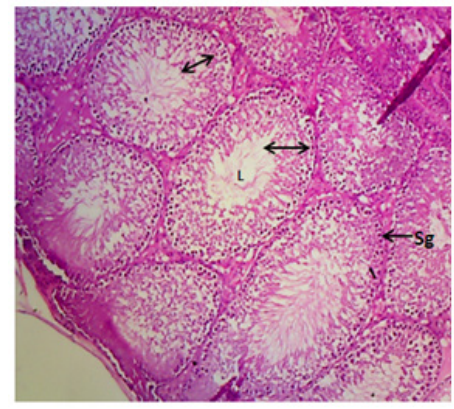

Figure 20: A section of the Testes: Male Medium Dose AA group (G3) Normal histology architecture of the testes (H \& E Stain) at magnification (X400) showing seminiferous tubules with spermatogonia (Sg), but with greatly reduced cellularity towards the lumen as the spermatogesis progresses (double end arrow) in many of the tubules.

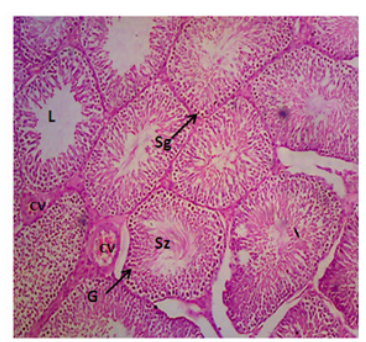

Figure 21: A section of the Testes: (High Dose AA group) Normal architecture of the testes (H \& E Stain) at magnification (X400) showing average sized seminiferous tubules with germinal cell layers, spermatogonia $(\mathrm{Sg})$, and evidence of spermatogenesis progressing from the basal germinal layer $(\mathrm{G})$ to mature spermatozoa $(\mathrm{Sz})$. Their tails are extending into the lumen $(\mathrm{L})$ and congested blood vessels $(\mathrm{CV})$ between tubules.

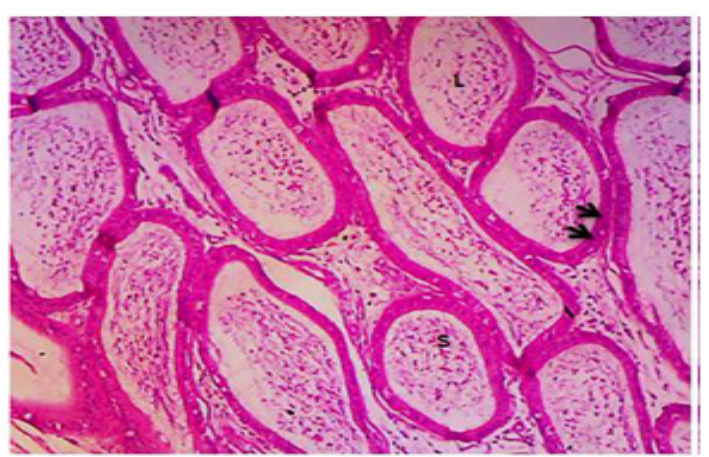

Figure 22: A section of the Epididymis (Unexposed Control) Histological section of epididymis (H \& E Stain) of animals in G1 at magnification (X400) showing normal architecture of the ductus epididymis with the lumen (L) filled with matured sperm cell (S). 


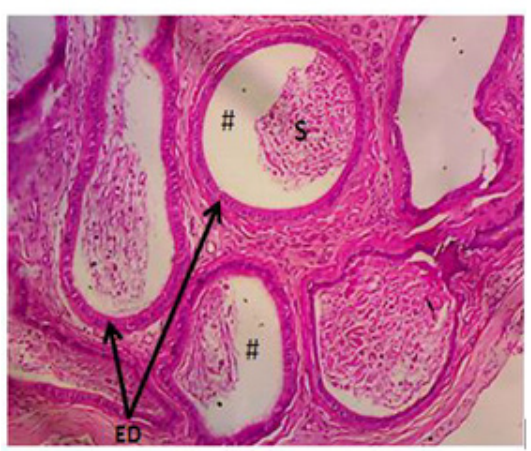

Figure 23: A section of the Epididymis (GV alone group) A section of the ductus epididymis (ED) (H \& E Stain) of animals in G2 at magnification (X400) showing a reduction in matured sperm cells (S), volume (L), and decreased spermatozoa number (\#) within the lumen.

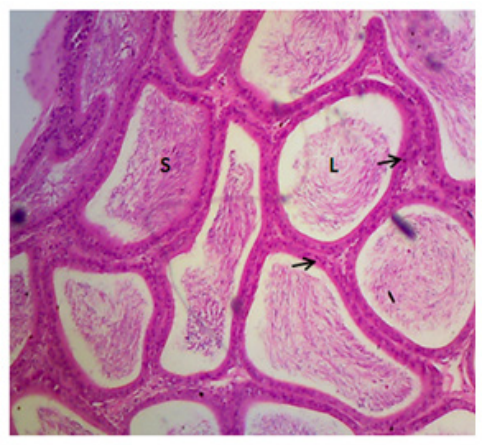

Figure 24: A section of the Epididymis (low Dose AA group) A longitudinal section of the ductus epididymis (H \& E Stain) at magnification (X400) showing the lumen (L) filled with matured sperm cell (S). The epididymal epithelia (arrow heads) were typical.

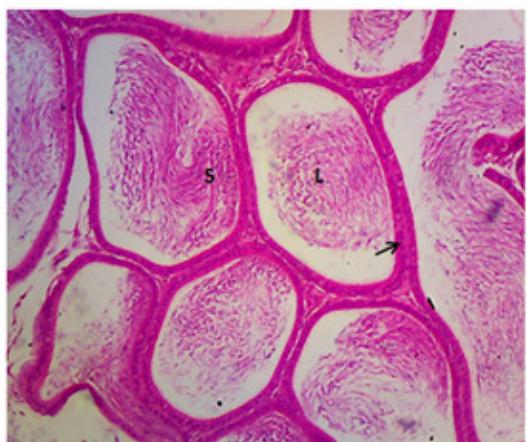

Figure 25: A section of the Epididymis (Medium Dose AA group) A longitudinal section of the ductus epididymis lumen (L) (H \& E Stain) at magnification (X400) filled with mature sperm cell (S). The epididymal epithelia (arrows) were typical.

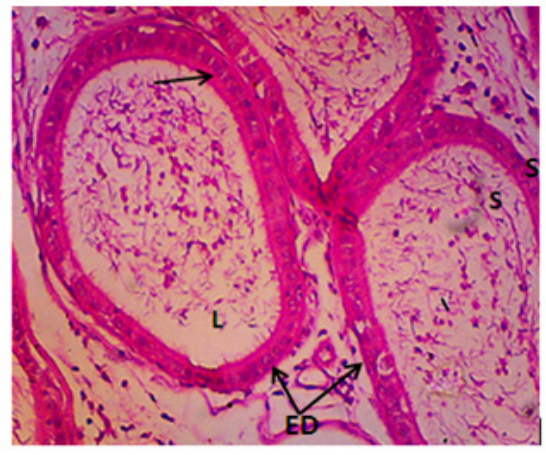

Figure 26: A section of Epididymis: (High Dose AA group) Normal histology architecture of the longitudinal section of ductus epididymis (ED) (H \& E Stain) at magnification (X400) with lumen (L) filled with mature sperm cell (S). The epididymal epithelia were typical (arrow heads). 


\section{Discussion}

The present study findings revealed that exposure to GV is associated with a significant alteration in OS status and reproductive system dysfunction in male and female rats. Accordingly, animals exposed to GV and concomitantly treated with different doses of AA showed dose-dependent beneficial or detrimental effects. For instance, serum levels of estrogen and progesterone in female animals were significantly higher in the group treated with low dose of AA. In contradistinction, male animals treated with the high dose of AA had the highest serum testosterone levels. Interestingly, the increase in serum progesterone and estrogen levels caused a corresponding decrease in serum levels of FSH and LH due to the negative feedback effect of the increased serum levels of the female sex hormones on the gonadotrophin-releasing hormone system. Also, improvement in the ovarian histomorphology following treatment with AA was more in the low-dose than the high-dose AA-treated female animals, and vice versa in the male group. Furthermore, serum CAT level increased, while serum MDA level decreased in the low dose AA-treated female animals. Whereas, male animals treated with a high dosage of AA had similar effects, demonstrating the gender-related heterogeneity in effects of AA. These findings are consistent with the results of a study conducted by Al-tib et al. [9], which showed that animals treated with a low dose of AA had a better ameliorative effect against potassium permanganate (KMno4)-induced ovarian dysfunction and OS including, improvement in the diameter of the ovary, corpus luteum, and graffian follicles as against the high dose AA-treated group with the least increment in the diameter of these structures. Besides the dose-dependent effect of AA against xenobiotic-induced ovarian dysfunction, the duration of treatment also showed an inverse relationship with the ovarian endpoints in previous studies. The acute treatment produced a higher protective effect on the ovary than chronic treatment. Accordingly, in a study to assess the action of AA on ovarian function of aging mice, Mohammad-Amin et al. [10] observed that acute treatment with AA produced a more beneficial effect on some reproductive endpoints than chronic treatment. Acute treatment of animals with AA (8 days) caused a better improvement in the volume of oocytes in the antral follicle, increased in the number of granulosa cells, primordial cells, secondary follicles, antral follicles, and primary follicles than those treated for 12 or 33 weeks. Also, a high dose of AA caused a decrease in serum superoxide dismutase (SOD) in granulosa cells. Similarly, Ismiyati et al. [11] studied the effect of combined $\mathrm{AA}$ and vitamin $\mathrm{E}$ against depot-medroxyprogesterone acetate (DMPA)-induced ovarian OS in rat and found that animals treated with the lowest dose of AA had a better ameliorative effect against DMPA-induced decrease in ovarian weight and SOD activity than the animals in the high dose AA treated group. Also, AA in its lowest dose significantly prevented DMPA-induced increase in MDA concentration in the ovarian tissue better than the group treated with the high dosage of AA. In the present study, female animals treated with a low dose of AA had the highest serum CAT and the least serum MDA, similar to males treated with the high dosage of AA. Furthermore, a study to assess the effect of AA on serum oestradiol in postmenopausal women reported that those who had the lowest plasma concentration of AA at baseline had the highest increment in plasma oestradiol level [12]. Also, one month of treatment with AA caused a significantly higher serum level of ovarian endpoints than three months of treatment. Convincing evidence indicates that the effect AA on plasma estrogen level was mediated by the synergistic antioxidant activities that provided a better antioxidant action than either of them alone, supporting the notion that antioxidants act as a cooperative network [13]. The contrary effects observed in the male group are similar to those of a plethora of research conducted to assess the action of $\mathrm{AA}$ on male reproductive performance in heterogeneous OS-related environments. Similarly, Sanghishetti et al. [14] found a better increase in indices of male reproductive function (testicular weight, seminiferous tubules weight, sperm count, and testosterone level) in the high dose AA-treated group than the low dose AA- treated group. Sperm concentration, sperm motility, and serum levels of testosterone and FSH were significantly higher in the high dose AA-treated group than the low dose AA treated and control groups in a study to assess the effect of AA on fertility parameters in male rats [15]. These findings are in good agreement with the bimodal characteristics of AA postulated by Schwartz [16]. A plausible explanation for the observed inverse correlation between the therapeutic dose and effect of AA on female reproductive endpoints may be because the female reproductive system naturally is endowed with efficient antioxidant systems. For instance, the ovaries have a rich and efficient antioxidant system made up of a non-enzymatic antioxidant (vitamin A, C, and E), and an enzymatic antioxidant (tripeptide glutathione, glutathione peroxidase (GPX), SOD, and CAT) [10,17-21], as well as estrogen the hormone of the female reproductive system has been shown to display an efficient antioxidant prowess. According to Bostanci et al. [13], there is a strong correlation between estrogen status and serum antioxidant capacity. These observations suggest that AA, when applied in low dosage, may provide a better protective effect against xenobiotic-induced reproductive dysfunction in females than the high dose treatment regimen. These could probably be because the low dosage of exogenous AA synergized with the rich ovarian antioxidant system to produce a synergistic/additive effect that plays a more antioxidant role than the high dosage of exogenous AA. Also, AA has a peak or maximum therapeutic serum/tissue concentration beyond which the efficacy declines. Earlier studies have observed that the ovary is the site of AA accumulation and turnover. The highest concentration of $\mathrm{AA}$ is present in the theca interna, granulosa, and corpus luteum. The follicular fluid has more AA than the serum, suggesting that the AA enters the follicular fluid against the concentration gradient by active transport $[22,23]$. Likely, the transport mechanism for AA is more effective at a low concentration of AA in the females and vice 
versa in the male animals. It is also a known fact that in a persistent OS as found in the present study, a critical serum concentration of ascorbate radicals is required to attend the highest effect, after which a steady decline ensues. This notion is in line with the theory of antioxidant paradox/or overuse that states that excessive use of antioxidants or an antioxidant combination may lead to reductive stress [24] that produces an effect similar to oxidative stress and is associated with some disease conditions including, cancer and cardiomyopathy [2]. Available evidence indicates that AA in very high concentrations can act as a pro-oxidant but behaves as an antioxidant in therapeutic doses [6]. Currently, there is a debate as to the best effective dose regimen of AA in terms of the amount and duration of intake for maintaining optimal health in humans. Accordingly, Chakraborthy et al. [6] studied the beneficial effects of AA in human health and disease and postulated that at high dosage, AA could behave as an antioxidant under physiological conditions, but switch over to a pro-oxidant under pathological condition, a view supported previously by Naidu [25].

\section{Conclusion}

Given the findings of the present study, it seems reasonable to opine that there is a gender difference in the effective dose of AA against xenobiotic-induced $O S$ and reproductive toxicity. Therefore, gender-related dose adjustment is required when using AA to manage OS-related male or female reproductive disorders. This novel observation is of clinical and public health importance as the wrong dosage or prolongs therapy with AA can worsen a preexisting xenobiotic -induced OS and reproductive toxicity.

\section{Acknowledgment}

None.

\section{Conflict of Interest}

No conflict of interest.

\section{References}

1. Caprino, Togna GI (1998) Potential health effects of gasoline and its constituents. A review of current literature (1990-1997) on toxicological data. Environ Health Perspect 106: 115-125.

2. Uboh FE, Akpanabiatu MI, Atangwho IJ, Alozie Y (2008) Exposure to gasoline vapors: A potential factor for atherosclerosis in male and female rats. J Pharmacol Toxicol 3(8): 600-609.

3. Arab SA, Nikravesh MR, Jalali M, Fazel A (2018) Evaluation of oxidative stress indices after exposure to malathion and protective effects of ascorbic acid in ovarian tissue of adult female rats. Electron Physician 10: 6789-95.

4. Soleimani Mehranjani M, Mansoori T (2016) Stereological study on the effect of vitamin $C$ in preventing the adverse effects of bisphenol A on rat ovary. Int J Reprod Biomed (Yazd) 14: 403-10.

5. Yarube IU, Ayo JO, Fatihu My (2014) Harmful effects of ascorbic acid and $\alpha$-tocopherol on male reproductive organs of rats chronically exposed to sodium nitrate. Journal of Mediana in the Tropics 16 (1): 5-8.

6. Chakraborthy A, Ramani P, Sherlin HJ, Premkumar P, Natesan A (2014) Anti-oxidant, and pro-oxidant activity of vitamin $\mathrm{C}$ in the ora environment. Indian Journal of Dental Research 25(4): 499-504.
7. Tietz NW (1986) Textbook of clinical chemistry. Saunders WB Cov Philadephia, pp.509-512.

8. Ekpenyong CE, Essiet U (2019) Cymbopogon citratus Stapf (DC) extract attenuates gasoline vapors-induced low-triiodothyronine syndrome oxidative stress and lipid peroxidation in rats. Trends in Phytochemical Research 3(5): 185-196.

9. Al-katib SR, AL-Azam AHA, Habeab SA (2012) The effect of vitamin C on the ovary of female white rats treated with kmno4. Histological and Physiological Study. Kufa Journal for Veterinary Medical Sciences 3(2): 1-16.

10. Mohammad-Amin Abdollahifar, Nahid Azad, Ensieh Sajadi, Zahra Shams Mofarahe, Fatemeh Zare, et al. (2019) Vitamin C restores ovarian follicular reservation in a mouse model of aging. Anat Cell Biol 52(2): 196-203.

11. Ismiyati A, Arsana IW, Hidayati DYN (2016) Protective effect of vitamin C and $\mathrm{E}$ on depot-medroxyprogesterone acetate-induced ovarian oxidative stress. Journal of toxicology.

12. Vihtamaki T, Parantainen J, Koivisto AM, Meta-ketela T, Tuimala R (2002) Oral ascorbic acid increase plasma oestradiol during postmenopausal hormone replacement therapy. Maturitas 42(2): 129-135.

13. Bostanci MS, Bayram M, Keskil ZA (2012) The effect of ascorbic acid on the estrogen/progesterone levels in the Isolated rabbit uterine muscle. J Clin Gynecol Obstet 1(4-5): 63-66.

14. Sanghishetti VP, Ghongane BB, Nayak BB (2014) Effect of vitamin C on male fertility in rats subjected to forced swimming stress J Clin DR 8(7): HC05-HC08.

15. Okon UA, Utuk II (2016) Ascorbic acid treatment elevates folliclestimulating hormone and testosterone plasma levels and enhances sperm quality in albino Wistar rats. Nigerian Medical Journal 59(1): 3136.

16. Schwartz JL (1996) The dual roles of nutrients as antioxidant and prooxidant. Their effects on tumor cell growth J Nutr 126: 1221s-1227s.

17. Sato EF, Kobuchi H, Edashige K, Takahashi M, Utsumi K, et al. (1992) Dynamic aspects of ovarian superoxide dismutase isozymes during the ovulatory process in the rat. FEBS Lett 303(2-3): 121-125.

18. Okatani Y, Morioka N, Wakatsuki A, Nakano Y, Sagara Y (1993) Role of the free radical-scavenger system in aromatase activity of the human ovary. Horm Res 39(1): 22-27.

19. Gardiner CS, Salmen JJ, Brandt CJ, Stover SK (1998) Glutathione is present in reproductive tract secretions and improves the development of mouse embryos after chemically induced glutathione depletion. Biol Reprod 59(2): 431-436.

20. Luderer U, Kavanagh TJ, White CC, Faustman EM (2001) Gonadotropin regulation of glutathione synthesis in the rat ovary. Reprod Toxicol 15: 495-504.

21. Tatone C, Carbone MC, Falone S, Aimola P, Giardinelli A Caserta D, et al. (2006) Age-dependent changes in the expression of superoxide dismutases and catalase are associated with ultrastructural modifications in human granulosa cells. Mol Hum Reprod 12(11): 655660.

22. Hofmann KD, Wagner F, Preibsch W, Koob G, Niedner W (1970) Ascorbic acid content of human ovary during vital and cycle phases in women. Zentraibl Gynakol 92(45): 1481-1484.

23. Veek L (1986) Atlas of the human Oocyte and early conception. Baltimore Williams \&Wilkins.

24. Henkel R, Sandhu IS, Agarwal A (2018) The excessive use of antioxidant therapy: A possible cause of male infertility Andrologia 51(1): e13162.

25. Naidu KA (2003) Vitamin C in human health and disease is still a mystery? An overview. Nutr J 2: 7. 